\title{
Reverse allostasis in biological systems: Minimal conditions and implications
}

\author{
Nasrollah Rezaei-Ghaleh ${ }^{\mathrm{a}, \mathrm{b}, *}$, Davood Bakhtiari $^{\mathrm{c}}$, Armin Rashidi $^{\mathrm{d}}$ \\ a Department of Structural Biology in Dementia, German Center for Neurodegenerative Diseases (DZNE), Von-Siebold-Strasse $3 a, 37075$ Göttingen, Germany \\ ${ }^{\mathrm{b}}$ Department for NMR-based Structural Biology, Max Planck Institute for Biophysical Chemistry, Am Fassberg 11, 37077 Göttingen, Germany \\ ${ }^{\mathrm{c}}$ Functional Imaging Section, Deutsches Primatenzentrum GmbH, Kellnerweg 4, 37077 Göttingen, Germany \\ ${ }^{\mathrm{d}}$ Division of Hematology, Oncology, and Transplantation, Department of Medicine, University of Minnesota, 420 Delaware Street SE, MMC 480, Minneapolis, \\ MN 55455, USA
}

\section{A R T I C L E I N F O}

\section{Article history:}

Received 15 December 2016

Revised 10 May 2017

Accepted 22 May 2017

Available online 26 May 2017

\section{Keywords:}

Homeostasis

Enzyme

Drug

Aggregation

Population

\begin{abstract}
A B S T R A C T
Biological control systems regulate the behavior of biological systems in a constantly changing environment. Homeostasis is the most widely studied outcome of biological control systems. Homeostatic systems maintain the system in its desired state despite variations in system parameters or the externallydetermined input rates of their constituents, i.e. they have zero or near zero steady state error. On the other hand, allostatic systems are not resistant against environmental changes and the steady state level of their controlled variables responds positively to the changes in their input rates. Little is known, however, on the existence and frequency of reverse allostatic systems, where the steady state value of the controlled variable correlates negatively with the input rate of that variable. In the present study, we derive the minimal conditions for the existence and local stability of reverse allostatic systems, and demonstrate in examples of metabolic, pharmacological, pathophysiological and ecological systems that the reverse allostasis requirements are relatively non-stringent and may be satisfied in biological systems more commonly than usually thought. The possible existence of reverse allostatic systems in nature and their counter-intuitive implications in physiological systems, drug treatment, ecosystem management, and biological control are explored and testable predictions are made.
\end{abstract}

(c) 2017 Elsevier Ltd. All rights reserved.

\section{Introduction}

In the face of continuously changing environments, living organisms have developed efficient control systems to maintain within appropriate ranges the variables critical to their survival (Northrop, 2000). The controlled variable may be physical, chemical or biological. Physical variables are exemplified by body temperature or blood pressure, chemical variables by blood glucose or cellular adenosine triphosphate (ATP) concentration, and biological variables by population of different cell types within tissues or population of different organisms within an ecosystem. The time-dependent level of the controlled variable is determined by its flux rates into and out of the biological system, as well as its generation and degradation or dissipation rates inside the system. When changes in the environment alter the relevant influx or efflux rates, the controlled variable undergoes a transient response

\footnotetext{
* Corresponding author at: German Center for Neurodegenerative Diseases (DZNE), Göttingen, c/o Max Planck Institute for Biophysical Chemistry, Am Fassberg 11, 37077 Göttingen, Germany.

E-mail address: nare@nmr.mpibpc.mpg.de (N. Rezaei-Ghaleh).
}

which gradually pushes it towards a new steady state level. For example, when dietary glucose intake is increased, the glucose control system triggers a complex series of hormonal, metabolic, and behavioral responses which regulate blood glucose in the new condition.

Biological control systems can be categorized on the basis of how the steady state value of their controlled variable depends on the input rate (the net balance of the influx and efflux rates) of that variable. Many control systems show zero steady state error, i.e. the steady state value of the controlled variable is independent of its input rate. Most homeostatic mechanisms, for example, include control systems with zero or near-zero steady state error (Wilkin, 1998). The blood concentration of critical ions such as potassium should be maintained within a remarkably narrow range for the organism to survive. Negative feedback loops are commonly found in such systems.

There also exist homeostatic systems with non-zero steady state errors. To be distinguished from homeostatic systems with zero steady state errors, systems with a positive steady state error are referred to as "allostatic systems". In these systems, the steady state value of the controlled variable rises with its increasing in- 
put rate. Allostatic systems are not necessarily detrimental or representative of pathology (Korte et al., 2007). Departure from the previously advantageous homeostatic value and settling into a new value when encountering conditions requiring new functionalities may in fact improve the fitness of the organism under stressful conditions in the short term (Lesne, 2008). The long-term effects of such chronic adaptive responses, however, are usually deleterious (Logan and Barksdale, 2008; Oken et al., 2015). Type 2 diabetes, for example, is the pathological outcome of a chronic glucose load via increased carbohydrate intake (Stumvoll et al., 2003).

Even though "reverse allostatic" systems in which the steady state value of the controlled variable negatively correlates with the input rate of that variable are theoretically possible, very few of them are known in physiological systems. Allostasis and reverse allostasis properties are unique in that they make the system extremely sensitive to the input-related parameters. "Resistance" (the ability of a system to withstand displacement by a disturbance in its environment) and "resilience" (the speed at which a system returns to its equilibrium following a perturbation) are two widely discussed concepts in ecosystem stability (Mori, 2016; Oliver et al., 2015). Allostatic and reverse allostatic systems show no resistance against perturbations that change the input rate of the variable for which the system has the non-zero steady state error property. In this article, we will derive minimal conditions for the existence of reverse allostatic systems, explore their local stability, and discuss situations where such systems could be found in nature. We provide examples of ecosystems which may show the reverse allostatic dynamics and explain how the "no resistance" property of such systems might be utilized in practice in the direction of interest. Also, in examples of simple metabolic and protein aggregation systems, we demonstrate how a particular disease condition may involve the system switch from one type to the other (e.g. from allostasis to reverse allostasis), and how knowledge about the behavior of the system would guide designing mechanistic therapeutic strategies.

\section{Theory}

Consider a dynamical system with one state variable, $x(t)$ and one state equation:

$d x / d t=I+f(x)$

where $I$ is a state-independent input rate of $x . I$ is an externally determined parameter of the system. $f(x)$ represents a statedependent rate of $x$, which depends on the value of $x$. At steady state, we have

$I=-f\left(x^{*}\right)$

where $x^{*}$ is the steady state value of $x$. For (1) to exhibit reverse allostasis, $f\left(x^{*}\right)$ must be an ascending function of $x^{*}$. This simple system, however, is not stable around its equilibrium point because if $x>x^{*}, f(x)>f\left(x^{*}\right)$ and therefore $d x / d t>0$, leading to further displacement of $x$ from $x^{*}$. A similar situation occurs if $x<x^{*}$. It is therefore necessary to increase the dimension of the system in order to have both reverse allostasis and local stability properties.

Now, let us consider a two-dimensional system with two state variables, $x$ and $y$, and two state equations:

$\frac{d x}{d t}=I_{1}+f(x, y)$
$\frac{d y}{d t}=I_{2}+g(x, y)$

where $I_{1}$ and $I_{2}$ are state-independent input rates of $x$ and $y$, respectively, and $f$ and $g$ represent state-dependent rates. At steady state we have:

$I_{1}=-f\left(x^{*}, y^{*}\right)$

$I_{2}=-g\left(x^{*}, y^{*}\right)$ where $x^{*}$ and $y^{*}$ are the steady state values of $x$ and $y$, respectively. Now, suppose that $I_{1}$ changes to $I_{1}+d I_{1}$ and $I_{2}$ remains constant $\left(d I_{2}=0\right)$. Then,

$d I_{1}=-(\partial f / \partial x) d x-(\partial f / \partial y) d y$

$d I_{2}=0=-(\partial g / \partial x) d x-(\partial g / \partial y) d y$

which gives:

$\frac{d y}{d x}=-\frac{(\partial g / \partial x)}{(\partial g / \partial y)}$

$\frac{d I_{1}}{d x}=-\frac{\partial f}{\partial x}-\frac{\partial f}{\partial y} \frac{d y}{d x}=-\frac{\partial f}{\partial x}+\frac{\partial f}{\partial y} \frac{(\partial g / \partial x)}{(\partial g / \partial y)}$

For a system to have the reverse allostasis property, we need $d x^{*} / d I_{1}<0$. From above, this condition occurs if and only if at $\left(x^{*}\right.$, $\left.y^{*}\right)$ we have:

$\frac{\left(\frac{\partial f}{\partial x}\right)\left(\frac{\partial g}{\partial y}\right)-\left(\frac{\partial f}{\partial y}\right)\left(\frac{\partial g}{\partial x}\right)}{\left(\frac{\partial g}{\partial y}\right)}>0$

According to the Hartman-Grobman theorem, the fixed point $\left(x^{*}, y^{*}\right)$ is locally stable for the system represented by the system (3) if and only if it is a stable fixed point for the linearization of the same system. Using the Jacobian matrix of the linearized system, the characteristic equation of the stability matrix of the linearized system is:

$\lambda^{2}-\left(\frac{\partial f}{\partial x}+\frac{\partial g}{\partial y}\right) \lambda+\left[\left(\frac{\partial f}{\partial x}\right)\left(\frac{\partial g}{\partial y}\right)-\left(\frac{\partial f}{\partial y}\right)\left(\frac{\partial g}{\partial x}\right)\right]=0$

The condition for local stability, i.e. the two eigenvalues having negative real parts, reduces to the following two criteria at fixed point $\left(x^{*}, y^{*}\right)$ :

$(\partial f / \partial x)(\partial g / \partial y)-(\partial f / \partial y)(\partial g / \partial x)>0$

$(\partial f / \partial x)+(\partial g / \partial y)<0$

From (7) and (9), and for (3) to have both the reverse allostasis property and local stability at its fixed point, it is necessary that at $\left(x^{*}, y^{*}\right)$ :

$\partial g / \partial y>0$

$\partial f / \partial x<-\partial g / \partial y<0$

Since $(\partial f / \partial x)(\partial g / \partial y)<0$, the first stability condition in (9) is satisfied only if

$(\partial f / \partial y)(\partial g / \partial x)<(\partial f / \partial x)(\partial g / \partial y)<0$

Finally, taking (9) to (11) into consideration, systems with either of the following properties:

A. $f$ and $g$ are respectively descending and ascending functions of both $x$ and $y$ (class-I)

B. Both $f$ and $g$ are descending functions of $x$ and ascending functions of $y$ (class-II)

would exhibit both reverse allostasis (for $x$ ) and local stability at equilibrium if:

$(\partial f / \partial x)<-(\partial g / \partial y)$

$(\partial f / \partial y)(\partial g / \partial x)<(\partial f / \partial x)(\partial g / \partial y)$

Fig. 1 shows a schematic representation of the two classes of reverse allostatic systems, highlighting that the common motif between them is the auto-inhibition of $x$ (the system variable with respect to which reverse allostasis is observed), auto-activation of $y$ and different signs of mutual hetero-interactions: if $x$ activates $y$, then $y$ should inhibit $x$ and vice versa.

\section{Results}

Now that we have determined the minimal requirements for the existence and local stability of reverse allostatic systems, we explore whether and how these conditions could be met in simple biological systems. 

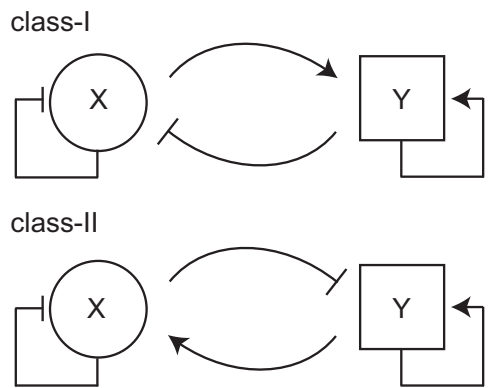

Fig. 1. Schematic representation of two classes of reverse allostatic systems, showing reverse allostatic behavior with respect to $\mathrm{X}$. $\mathrm{X}->\mathrm{Y}$ indicates " $\mathrm{X}$ activates $\mathrm{Y}$ " and $\mathrm{X}-\mathrm{I} Y$ indicates " $\mathrm{X}$ inhibits $\mathrm{Y}$ ".

\subsection{Metabolic systems}

For a simple biochemical system with reverse allostasis and local stability, we consider a system of two metabolites, $x$ and $y$, which are generated with externally determined constant rates and degraded enzymatically. The enzymatic degradation of the two metabolites is assumed to be coupled: $y$ is an activator of the enzymatic degradation of $x$, while $x$ inhibits the enzymatic degradation of $y$ (in a non-competitive manner). In addition, the enzymatic degradation of $y$ is supposed to follow a substrate-inhibition scheme, indicating that the enzymatic degradation rate rises to a maximum then decreases as $y$ concentration increases. This condition is central to the behavior of our system. The dynamics of this system can be described as:

$$
\begin{aligned}
& \frac{d x}{d t}=I_{1}-\frac{\alpha_{1} x \cdot\left(1+\frac{y}{\mu_{12}}\right)}{\left(K_{1}+x\right)} \\
& \frac{d y}{d t}=I_{2}-\frac{\alpha_{2} y}{\left(K_{2}+y+\frac{y^{2}}{\mu_{22}}\right) \cdot\left(1+\frac{x}{\mu_{21}}\right)}
\end{aligned}
$$

where $\alpha_{\mathrm{i}}$ and $K_{\mathrm{i}}$ are the maximum rate and constants for the enzymatic degradation of the metabolites and $\mu_{\mathrm{ij}}$ is the effect of $j$ on the enzymatic degradation of $i(i, j=1$ or 2 , for $x$ or $y)$. Fig. 2 illustrates the dynamical behavior of this system, exhibiting both reverse allostasis and local stability. As shown in Fig. 2, the gradual increase in the input rate of $x\left(I_{1}\right)$ leads to a decrease in the steady state value of its concentration (i.e. reverse allostasis) and the system remains stable around the steady state point. Notably, this system is a class-I reverse allostatic system and the trajectories revolve around the fixed point in counter-clockwise direction.

\subsection{Pharmacological systems}

Drugs and exogenous compounds (xenobiotics) frequently exhibit complex mutual interactions at the pharmacokinetic level, e.g. they can affect the metabolic rate of their own and other drugs by inducing or inhibiting the responsible enzymes. If two drugs, $x$ and $y$, are metabolized by the same enzyme, which is induced by $x$ but inhibited by $y$, then the time-dependent changes of the blood level of the two drugs may be described by the following equations:

$$
\begin{aligned}
& \frac{d x}{d t}=I_{1}-\frac{\alpha_{1} x\left(1+\frac{x}{\mu_{1}}\right)}{\left(K_{1}+x\right)\left(1+\frac{y}{\mu_{2}}\right)} \\
& \frac{d y}{d t}=I_{2}-\frac{\alpha_{2} y \cdot\left(1+\frac{x}{\mu_{1}}\right)}{\left(K_{2}+y\right)\left(1+\frac{y}{\mu_{2}}\right)}
\end{aligned}
$$

where $\alpha_{\mathrm{i}}$ and $K_{\mathrm{i}}$ represent maximum rate and constants for the enzymatic metabolism of these two drugs and $\mu_{\mathrm{i}}$ represents the modulatory effect of metabolite $i$ on the involved enzyme $(i, j=1$ or 2 , for $x$ or $y$ ). Since $x$ induces degradation of $y$ (and therefore decreases its blood level) while drug $y$ has an opposite effect on $x$, the system described by Eq. 14 could behave as a class-II reverse allostatic system (see Fig. 1). As illustrated in Fig. 3, with the right choice of the above parameters, the two-drug system exhibits stable reverse allostasis behavior. The trajectories of this system revolve around the fixed point in clockwise direction, contrary to the counter-clockwise direction of class-I reverse allostatic trajectories.

\subsection{Protein aggregation systems}

Another example of reverse allostatic systems is found in coupled protein aggregation systems in neurodegenerative diseases. Suppose that we have two proteins, $x$ and $y$, in aggregate forms (e.g. amyloid- $\beta$ and tau protein, respectively, in Alzheimer disease). $x$ induces the formation of $y$, while $y$ inhibits $x$ aggregation. The protein aggregate $x$ is normally degraded according to a MichaelisMenten-like enzymatic kinetic, and the enzymatic degradation of protein aggregate $y$ is inhibited when it is accumulated. The concentration evolution of the two protein aggregates is then governed by the following equations:

$$
\begin{aligned}
& \frac{d x}{d t}=I_{1}+\left(\frac{\mu_{12}{ }^{n}}{\mu_{12}{ }^{n}+y^{n}}\right) \beta_{1} x-\frac{\alpha_{1} x}{\left(K_{1}+x\right)} \\
& \frac{d y}{d t}=I_{2}+\left(\frac{x^{n}}{\mu_{21}{ }^{n}+x^{n}}\right) \beta_{2} y-\frac{\alpha_{2} y}{\left(K_{2}+y+\frac{y^{2}}{\mu_{22}}\right)}
\end{aligned}
$$

where $\alpha_{\mathrm{i}}$ and $\beta_{\mathrm{i}}$ are maximum rates of enzymatic degradation and production of these two protein aggregates, respectively, $K_{\mathrm{i}}$ are corresponding constants for the enzymatic degradation of these two protein aggregates, and $\mu_{\mathrm{ij}}$ represents the effect of protein $j$ on the production or enzymatic degradation of protein $i(i, j=1$ or 2 , for $x$ or $y$ ). With the right choice of parameters, this system manifests reverse allostatic behavior and local stability. Supplementary Information Figure S1 illustrates an example of such systems with a class-I reverse allostatic behavior. The system trajectories revolve around the fixed point in counter-clockwise manner.

\subsection{Population dynamics}

We explore the fourth example of reverse allostatic systems in population dynamics. Suppose two species, $x$ and $y$, living together and their population evolve according to a logistic-type dynamics:

$$
\begin{aligned}
& f(x, y)=\alpha_{1} \cdot x \cdot\left(1-\frac{x}{\gamma_{1}}\right) \\
& g(x, y)=\alpha_{2} \cdot y \cdot\left(1-\frac{y}{\gamma_{2}}\right)
\end{aligned}
$$

where

$\alpha_{1}=\mu_{12} y+\beta_{1}$

$\alpha_{2}=\mu_{21} x+\beta_{2}$

are Malthusian parameters (maximum rates of population growth) and the effect of species $j$ on species $i$ is represented by the interaction parameters $\mu_{\mathrm{ij}}$. If both $\mu_{12}$ and $\mu_{21}$ are negative, the system describes competition between two species, if both are positive, the two species benefit symbiosis, and if $\mu_{12}$ is negative but $\mu_{21}$ is positive, there is a prey-predator like interaction between them (with $x$ as a prey). The two populations are subject to constant (net) input rates, $I_{1}$ and $I_{2}$ (as in Eq. 3) and parameters $\gamma_{1}$ and $\gamma_{2}$ represent the intrinsic limiting values of two populations in the absence of input rates, i.e. when $I_{1}=I_{2}=0$. In such systems, if we limit ourselves to the steady states in which the two populations are below their intrinsic limiting values, then it could be shown that the stability condition in (11) is not met unless $\mu_{12}$ 

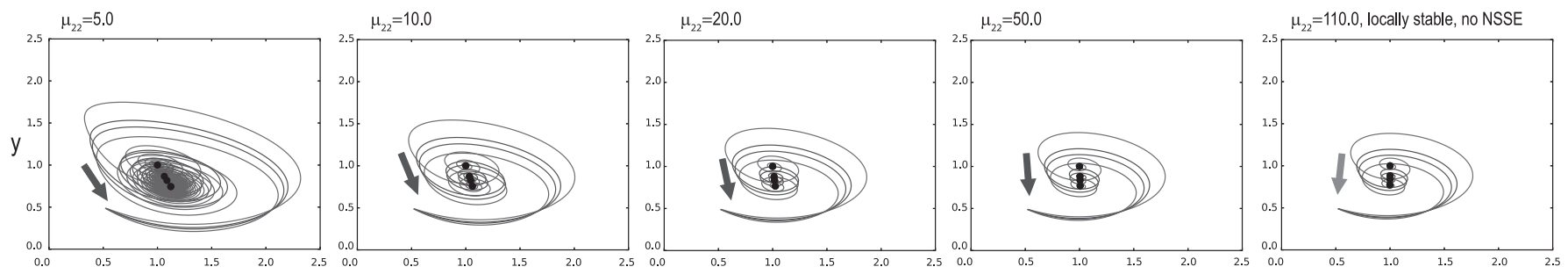

$$
\begin{aligned}
& l_{1}=13.6, l_{2}=5.51 \\
& -l_{1}=13.1, l_{2}=5.51 \\
& l_{1}=12.9, l_{2}=5.51
\end{aligned}
$$
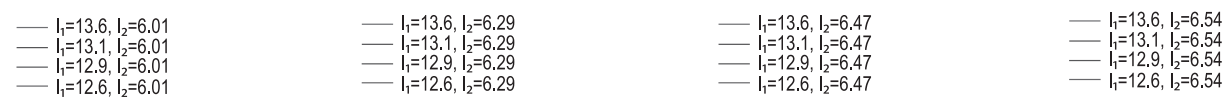

$$
\mu_{22}=5.0
$$

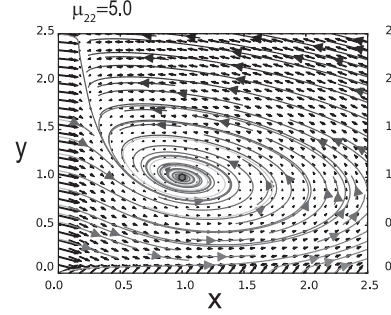

$\mu_{22}=10.0$

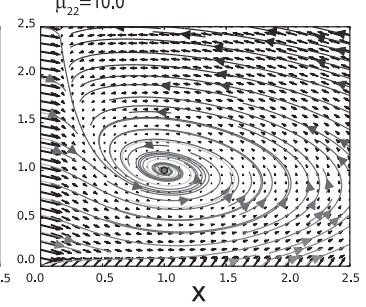

$\mu_{22}=20.0$

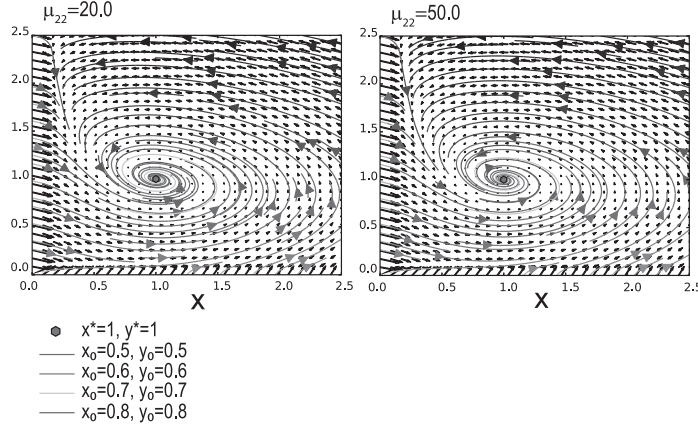

$L_{22}=110.0$, locally stable, no NSSE

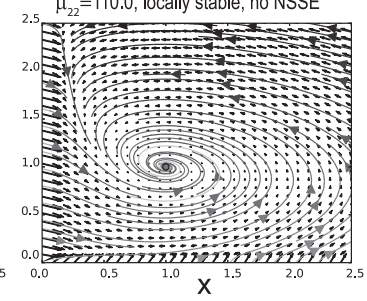

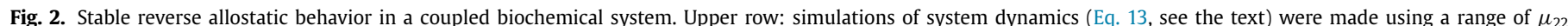

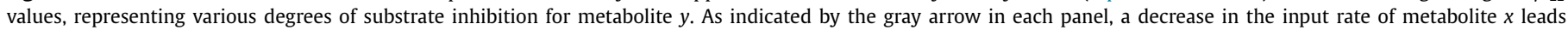

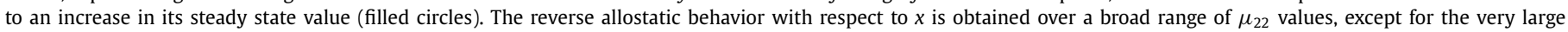

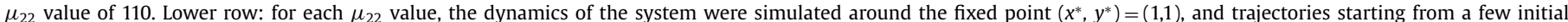

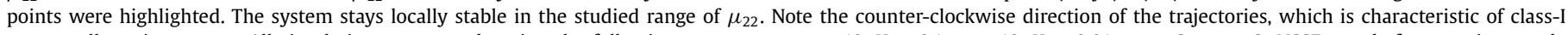

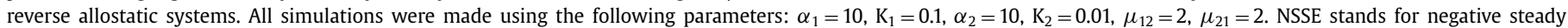
state error.
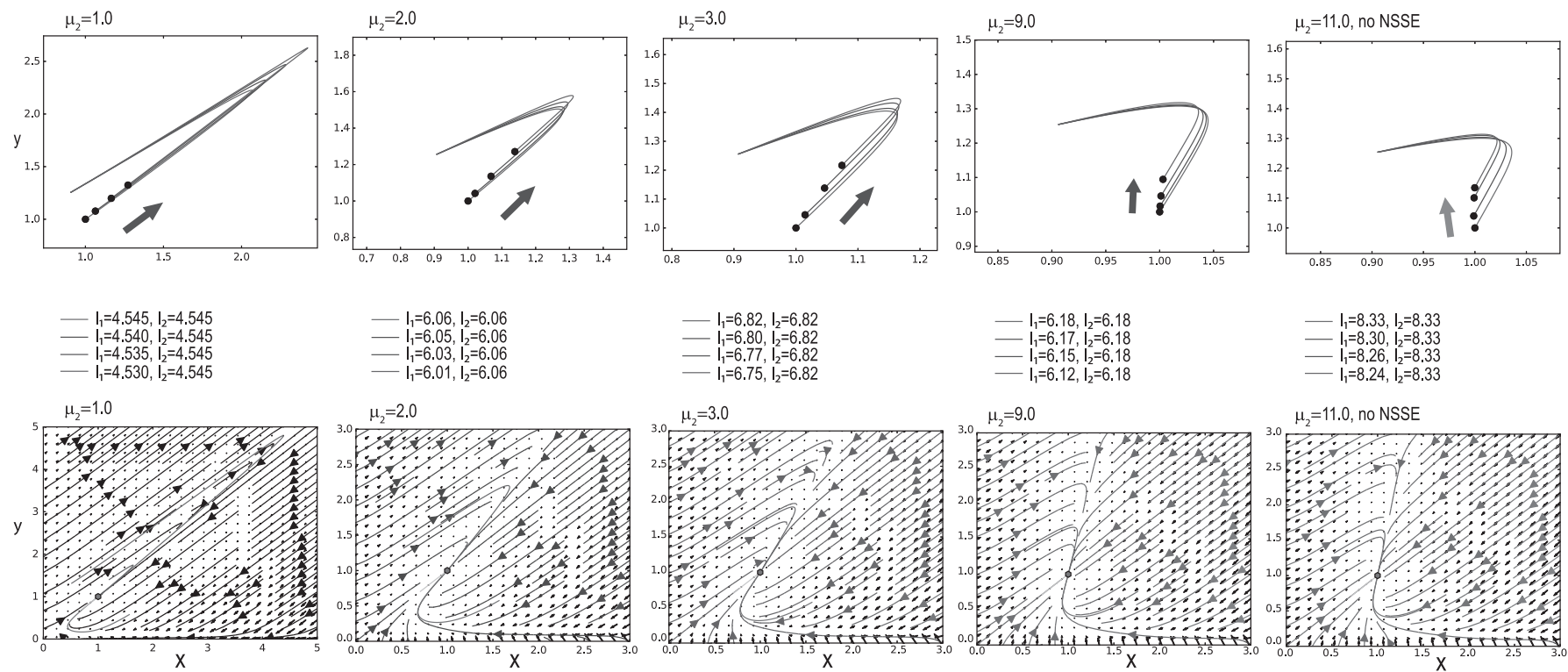

$l_{1}=8.33, l_{2}=8.33$
$l_{1}=8.30, l_{2}=8.33$

- $l_{1}=6.77, l_{2}=6.82$

- $\mathrm{l}_{1}=6.15, \mathrm{l}_{1}=6.18$

- $\left.\right|_{1}=8.26, l_{2}=8.33$
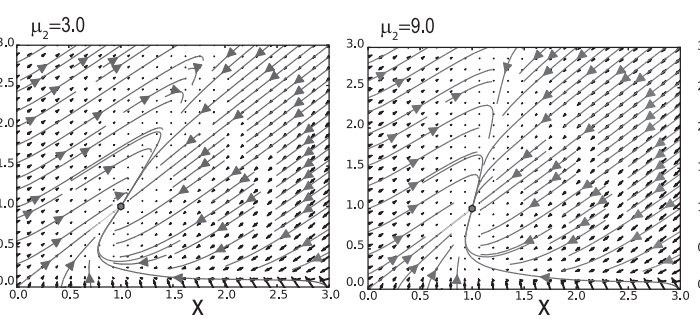

$\mu_{2}=11.0$, no NSSE

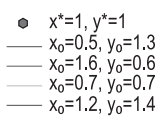

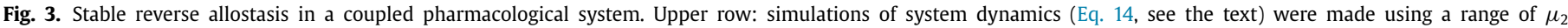

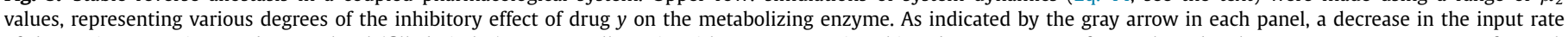

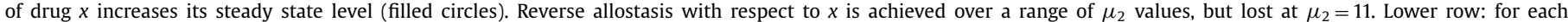

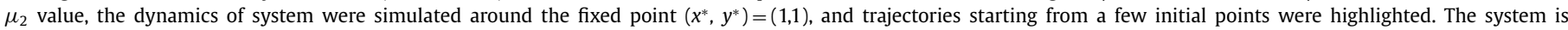

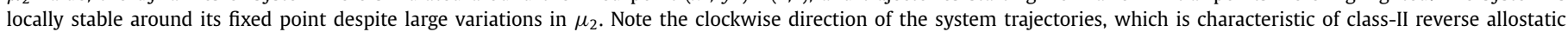
systems. All simulations were made using the following parameters: $\alpha_{1}=5, K_{1}=0.1, \alpha_{2}=5, K_{2}=0.1, \mu_{1}=1$. NSSE stands for negative steady state error. 
and $\mu_{21}$ have different signs, i.e. a prey-predator like interaction is essential for the system to be stable. Supplementary Information Figure S2 illustrates the dynamical behavior of an example of such systems which exhibit both the reverse allostatic behavior and local stability. A crucial feature of this example is the negative sign of $\beta_{2}$, indicating a "grouping effect" for species $y$. In this system, the gradual increase in the input rate of species $1\left(I_{1}\right)$ leads to a decrease in the steady state value of its population that is the reverse allostatic behavior and the system remains stable around the steady state for a relatively wide range of the varied parameter, $\beta_{1}$. In addition, this system is again a class-I reverse allostatic system and its trajectories move around the fixed point in counterclockwise direction.

\section{Discussion}

We explored whether the steady state value of a system variable, $x$, can change in the opposite direction of its externally determined input rate, i.e. reverse allostasis with respect to $x$. It was shown that one-dimensional systems cannot have the reverse allostasis property along with local stability. To behave as a stable reverse allostatic system with respect to $x$, the system requires to be at least two-dimensional and $x$ to be mutually coupled with a second variable $y$. We derived the minimal conditions for existence and local stability of two-dimensional reverse allostatic systems. We demonstrated in some simple biological systems that these requirements can be satisfied, provided that the coupled variable $y$ changes with a rate that increases when $y$ itself increases. This latter condition, as non-trivial as it seems to be, is indeed frequently encountered in biological systems.

Complex systems of enzyme reactions can be modeled using networks of coupled ordinary differential equations (ODEs) (Chen et al., 2010; Wong et al., 2015). When spatial distribution of enzymes is a relevant factor, e.g. in sequential enzyme reactions, transport and diffusion of intermediate reactants between consecutive enzymes are modeled using partial differential equations (Eun et al., 2014). In our example of the coupled two-enzyme system described above, the existence of stable reverse allostatic dynamics with respect to a metabolite is crucially dependent on the substrate inhibition of the enzyme responsible for degradation of its coupled metabolite. The property indicates that the enzyme activity increases to a maximum then decreases when the substrate concentration increases. It has been shown for several enzymes that substrate inhibition has important biological functions (Reed et al., 2010), and it is estimated that around 20\% of enzymes show some level of substrate inhibition (Chaplin and Bucke, 1990; $\mathrm{Wu}, 2011$ ). We speculate that the reverse allostasis behavior may exist for metabolites coupled to the substrate of enzymes with substrate inhibition. Since some enzymes involved in neurodegenerative diseases are subject to substrate inhibition (Reed et al., 2010), the potential existence of reverse allostasis related to these enzymes may have important implications in treatment of neurodegenerative diseases. In such cases, if a decrease in the level of the coupled metabolite is desired, the input rate of that metabolite should be increased (rather than decreased, as intuitively thought).

Some pharmacological systems with complex drug-drug interactions provide an important example of reverse allostatic systems. A prototypical example is cytochrome P450 (CYP450), the main metabolizing system in the liver responsible for biotransformation of drugs and xenobiotics. CYP450 has several isoforms (e.g. 3A4, 2D6 and 2C9) each of which metabolizes a specific group of exogenous compounds. Some compounds activate or inhibit the specific CYP450 isoform responsible for their own metabolism. If two drugs metabolized by the same CYP450 isoform have different modulatory effects on their CYP40, i.e. one of them activates while the other one inhibits it, the coupled drug system will be capable of exhibiting reverse allostasis. If the two drugs are administered simultaneously, a counter-intuitive reverse allostatic behavior may be detectable for one of the two drugs. One potential example of such systems is the pair of carbamazepine (an anticonvulsant drug) and verapamil (an antihypertensive drug), which are both metabolized by the 3A4 isoform. While carbamazepine induces 3A4 activity and thereby promotes degradation of itself and verapamil (Kang et al., 2008), verapamil inhibits 3A4 activity and has opposite effects (Zhou, 2008). With the right set of parameter values, the coupled carbamazepine-verapamil system could exhibit class-II reverse allostatic behavior with respect to carbamazepine: the higher dose of carbamazepine will lower its steady state level. Another example is the pair of phenobarbital (an anticonvulsant drug) and warfarin (an anticoagulant drug), which are metabolized by 2C9 and respectively induce or inhibit its activity (Greenblatt and von Moltke, 2005). Under the appropriate conditions, this coupled system could also behave as a class-II reverse allostatic system with respect to phenobarbital: the lower dose of phenobarbital will increase its steady state level. In case of anticonvulsant drugs, low or high drug levels may cause convulsions or comatose states, therefore it is crucial to consider the possibility of reverse allostatic behavior in such coupled systems. The two examples described above are well-known instances of significant drug-drug interactions and their simultaneous use is usually avoided in medical practice. However, our study suggests that the requirements for reverse allostasis are relatively non-stringent and may be met in many coupled drug-drug and drug-xenobiotic systems. As a consequence, counterintuitive dose-response behaviors may arise for many drugs and be more prevalent than is usually thought.

Coupled protein aggregation systems exemplify another class of biological systems with potential of stable reverse allostatic behavior. Neurodegeneration-related protein aggregation is frequently mutually coupled, a prototypical example of which is the coupling between amyloid- $\beta$ and tau protein aggregation in Alzheimer disease where each protein aggregate could influence the rate of generation or degradation of the other protein aggregate (Nisbet et al., 2015). For example, it is believed that amyloid- $\beta$ aggregation increases tau protein aggregation, while tau protein aggregation at a later stage induces neurodegeneration and therefore indirectly reduces the level of amyloid- $\beta$ and its aggregation. A key requirement for reverse allostasis in such systems is that the proteolytic degradation of an aggregated protein is reduced when it is accumulated, which could be easily fulfilled in our example when susceptibility of tau protein aggregates to proteases decreases at higher assembly states of tau protein, or even further, the proteolytic machinery of neurons is impaired in response to tau protein aggregation. Since the sufficient conditions for the existence of reverse allostasis in such systems are not very stringent, we hypothesize that reverse allostasis may exist in the course of protein aggregation-related diseases, at least in some phases. Counterintuitive phenomena may arise as a result. In Alzheimer disease, for example, the steady state level of amyloid- $\beta$ aggregates in a specific brain compartment may decrease when higher level of diffusible amyloid- $\beta$ aggregates are imported into that compartment from other brain regions.

In addition to the organism-level physiological and pathological systems, reverse allostatic systems may have important implications at the level of populations. In our example of a simple predator-prey system, we demonstrated that reverse allostasis with respect to the prey species emerges if the predator follows a strong "group effect". Counterintuitively and because of the intriguing dynamics of the system, the steady state frequency of organism $x$ decreases with increasing rates of their immigration. If for environmental concerns one is interested in lowering the frequency of $x$, one should try to promote rather than block its influx. 
A similar scenario may occur when there is a mixed population of two pathogens, say $x$ and $y$ in the body, with a constant influx. Each pathogen releases certain substances (e.g. products of metabolism, toxins, and growth factors) in the body. If these substances negatively impact survival of $x$, but are beneficial for $y$ (e.g. substances used as nutrients), then $f$ will decrease while $g$ will increase with increases in either $x$ or $y$, as required for class-I reverse allostatic systems. If the reverse allostatic behavior with respect to $x$ arises, the steady state density of microorganism $x$ will be a direct (rather than inverse, as intuitively thought) correlate of the immune barriers against $x$. From an evolutionary perspective, if $x$ is a more serious threat than $y$ to survival, the selection pressure for immune barriers against $x$ may become relaxed during the course of evolution in order to lower the steady state of $x$ in the body.

The possible existence of pathophysiological reverse allostatic systems suggests a new conceptual framework to understand disease versus healthy states not only in terms of the steady state or dynamical behavior around the fixed points, but also in terms of response to changes in the input rates, i.e. whether they are completely resistant against changes in the input rates (homeostatic system) or behave as an allostatic or a reverse allostatic system. Further classification of disease states on the basis of their allostatic or reverse allostatic behavior has significant therapeutic implications. In addition, it may offer new ways to interpret and explain the claims and findings of a complementary clinical school known widely as homeopathy. According to this school, in order to treat a disorder in a physiological system, a force should be applied upon the system in the same direction as the disorder (Bell et al., 2013; D'Huyvetter and Cohrssen, 2002; Jonas et al., 2003), e.g. if the blood glucose is above normal, a medication which normally would elevate the blood glucose should be prescribed. This medication can result in lowering the blood glucose in a hyperglycemic patient. The present study offers a potential explanation for cases in which disease behaves as a reverse allostatic system. However, one should notice that the conditions we derived only guarantee local, and not necessarily global, stability of the steady state. This means that large changes in the input rate of the variable of interest do not necessarily result in the system moving to its new stable fixed point. Also, the apparent rarity of reverse allostatic conditions in physiological systems limits the applicability of homeopathy.

In conclusion, we derived the minimal conditions for a twodimensional dynamical system to exhibit stable reverse allostatic behavior and provided a number of biological examples where such systems may be found. Reverse allostasis may have implications in areas other than biology, e.g. economy and sociology. Future studies may look into these fields, explore higher dimensional systems, or consider time-dependent influx rates.

\section{Author contribution}

NR-G conceived the project, developed the theory, analyzed the models and wrote the manuscript. DB and AR contributed to model evaluation and manuscript writing.

\section{Acknowledgements}

NR-G acknowledges a Landahl travel grant from the Society for Mathematical Biology. This research did not receive any specific grant from funding agencies in the public, commercial, or not-forprofit sectors.

\section{Supplementary materials}

Supplementary material associated with this article can be found, in the online version, at doi:10.1016/j.jtbi.2017.05.025.

\section{References}

Bell, I.R., Koithan, M., Brooks, A.J., 2013. Testing the nanoparticle-allostatic crossadaptation-sensitization model for homeopathic remedy effects. Homeopathy 102 (66-81). doi:10.1016/j.homp.2012.10.005.

Chaplin, M., Bucke, C., 1990. Enzyme Technology. Cambridge University Press, Cambridge.

Chen, W.W., Niepel, M., Sorger, P.K., 2010. Classic and contemporary approaches to modeling biochemical reactions. Genes Dev. 24, 1861-1875. doi:10.1101/gad. 1945410.

D'Huyvetter, K., Cohrssen, A., 2002. Homeopathy. Prim. Care 29, 407-418 viii.

Eun, C., Kekenes-Huskey, P.M., Metzger, V.T., McCammon, J.A., 2014. A model study of sequential enzyme reactions and electrostatic channeling. J. Chem. Phys. 140, 105101. doi: $10.1063 / 1.4867286$

Greenblatt, D.J., von Moltke, L.L., 2005. Interaction of warfarin with drugs, natural substances, and foods. J. Clin. Pharmacol. 45, 127-132. doi:10.1177/ 0091270004271404.

Jonas, W.B., Kaptchuk, T.J., Linde, K., 2003. A critical overview of homeopathy. Ann. Intern. Med. 138, 393-399.

Kang, P., Liao, M., Wester, M.R., Leeder, J.S., Pearce, R.E., Correia, M.A., 2008. CYP3A4-Mediated carbamazepine (CBZ) metabolism: formation of a covalent CBZ-CYP3A4 adduct and alteration of the enzyme kinetic profile. Drug Metab. Dispos. 36, 490-499. doi:10.1124/dmd.107.016501.

Korte, S.M., Olivier, B., Koolhaas, J.M., 2007. A new animal welfare concept based on allostasis. Physiol. Behav. 92, 422-428. doi:10.1016/j.physbeh.2006.10.018.

Lesne, A., 2008. Robustness: confronting lessons from physics and biology. Biol. Rev. 83, 509-532. doi:10.1111/j.1469-185X.2008.00052.x.

Logan, J.G., Barksdale, D.J., 2008. Allostasis and allostatic load: expanding the discourse on stress and cardiovascular disease. J. Clin. Nurs. 17, 201-208. doi:10. 1111/j.1365-2702.2008.02347.x.

Mori, A.S., 2016. Resilience in the studies of biodiversity-ecosystem functioning. Trends Ecol. Evol. 31 (87-9). doi:10.1016/j.tree.2015.12.010.

Nisbet, R.M., Polanco, J.C., Ittner, L.M., Gotz, J., 2015. Tau aggregation and its interplay with amyloid-beta. Acta Neuropathol. 129, 207-220. doi:10.1007/ s00401-014-1371-2.

Northrop, R.B., 2000. Endogenous and Exogenous Regulation and Control of Physiological Systems. Chapman \& Hall/CRC, Massachusetts.

Oken, B.S., Chamine, I., Wakeland, W., 2015. A systems approach to stress, stressors and resilience in humans. Behav. Brain Res. 282, 144-154. doi:10.1016/j.bbr.2014. 12.047.

Oliver, T.H., Heard, M.S., Isaac, N.J., Roy, D.B., Procter, D., Eigenbrod, F., Freckleton, R., Hector, A., Orme, C.D., Petchey, O.L., Proenca, V., Raffaelli, D., Suttle, K.B., Mace, G.M., Martin-Lopez, B., Woodcock, B.A., Bullock, J.M., 2015. Biodiversity and resilience of ecosystem functions. Trends Ecol. Evol. 30 (673-84). doi:10.1016/j.tree.2015.08.009.

Reed, M.C., Lieb, A., Nijhout, H.F., 2010. The biological significance of substrate inhibition: a mechanism with diverse functions. Bioessays 32, 422-429. doi:10. 1002/bies.200900167.

Stumvoll, M., Tataranni, P.A., Stefan, N., Vozarova, B., Bogardus, C., 2003. Glucose allostasis. Diabetes 52, 903-909.

Wilkin, T.J., 1998. Endocrine feedback control in health and disease. In: Bittar, E.E., Bittar, N. (Eds.), The Principles of Medical Biology. JAI Press, Greenwich, pp. $1-28$.

Wong, M.K., Krycer, J.R., Burchfield, J.G., James, D.E., Kuncic, Z., 2015. A generalised enzyme kinetic model for predicting the behaviour of complex biochemical systems. FEBS Open Bio 5 (226-39). doi:10.1016/j.fob.2015.03.002.

$\mathrm{Wu}$, B., 2011. Substrate inhibition kinetics in drug metabolism reactions. Drug Metab. Rev. 43, 440-456. doi:10.3109/03602532.2011.615320.

Zhou, S.F., 2008. Drugs behave as substrates, inhibitors and inducers of human cytochrome P450 3A4. Curr. Drug Metab. 9, 310-322. 